\title{
Erratum to: Orally administered multispecies probiotic formulations to prevent uro-genital infections: a randomized placebo-controlled pilot study
}

\author{
Valerio Mezzasalma ${ }^{1}$ Enrico Manfrini ${ }^{1} \cdot$ Emanuele Ferri $^{1} \cdot$ Marco Boccarusso $^{1}$. \\ Patrizia Di Gennaro $^{1} \cdot$ Irene Schiano $^{2} \cdot$ Angela Michelotti $^{2} \cdot$ Massimo Labra $^{1}$
}

Published online: 23 December 2016

(C) Springer-Verlag Berlin Heidelberg 2016

\section{Erratum to: Arch Gynecol Obstet \\ DOI 10.1007/s00404-016-4235-2}

The author would like to correct the error in the online published article:

In Materials and methods under sub heading Strains, probiotic formulations and culture conditions, in paragraph 3 , sentence 2 should read as:

The composition of the probiotic mix F_1 was as follows: $1 \times 10^{9} \mathrm{CFU}$ L. acidophilus PBS066 (40 mg as lyophilized), $1 \times 10^{9} \mathrm{CFU}$ L. reuteri $\mathrm{PBS} 072$ (30 mg as lyophilized), $320 \mathrm{mg}$ inulin, $5 \mathrm{mg}$ silica, $5 \mathrm{mg}$ talc. The F_2 composition was as follows: $1 \times 10^{9} \mathrm{CFU}$ L. plantarum PBS067 (12 mg as lyophilized), $1 \times 10^{9} \mathrm{CFU} L$. rhamnosus PBS070 (20 mg as lyophilized), $1 \times 10^{9} \mathrm{CFU}$ B. animalis subsp. lactis PBS075. (60 $\mathrm{mg}$ as lyophilized), $298 \mathrm{mg}$ inulin, $5 \mathrm{mg}$ silica, $5 \mathrm{mg}$ talc. Placebo (F_3) composition was as follows: $390 \mathrm{mg}$ inulin, $5 \mathrm{mg}$ silica, $5 \mathrm{mg}$ talc.

The online version of the original article can be found under doi: 10.1007/s00404-016-4235-2.

Patrizia Di Gennaro

patrizia.digennaro@unimib.it

1 Department of Biotechnology and Biosciences, University of Milano-Bicocca, Piazza della Scienza 2, 20126 Milan, Italy

2 Farcoderm Srl, Via Angelini 21,

27028 San Martino Siccomario, Pavia, Italy 